\title{
Recent results from kaon physics
}

\author{
Giuseppe Ruggiero*挤 \\ Lancaster University \\ E-mail: giuseppe.ruggiero@cern.ch
}

The $\pi v v$ is one of the theoretically cleanest meson decay where to look for indirect effects of new physics complementary to LHC searches. The NA62 experiment at CERN SPS is designed to measure the branching ratio of the $K^{+} \rightarrow \pi^{+} v \bar{v}$ decay with $10 \%$ precision. NA62 took data in 2015 and 2016 reaching the Standard Model sensitivity. The KOTO experiment in Japan is investigating the decay $K_{L} \rightarrow \pi^{0} v \bar{v}$ reaching a higher background suppression. Both experiments are reviewed, and recent results and prospects are summarised.

EPS-HEP 2017, European Physical Society conference on High Energy Physics

5-12 July 2017

Venice, Italy

${ }^{*}$ Speaker.

${ }^{\dagger}$ for the NA62 Collaboration: R. Aliberti, F. Ambrosino, R. Ammendola, B. Angelucci, A. Antonelli, G. Anzivino, R. Arcidiacono, M. Barbanera, A. Biagioni, L. Bician, C. Biino, A. Bizzeti, T. Blazek, B. Bloch-Devaux, V. Bonaiuto, M. Boretto, M. Bragadireanu, D. Britton, F. Brizioli, M.B. Brunetti, D. Bryman, F. Bucci, T. Capussela, A. Ceccucci, P. Cenci, V. Cerny, C. Cerri, B. Checcucci, A. Conovaloff, P. Cooper, E. Cortina Gil, M. Corvino, F. Costantini, A. Cotta Ramusino, D. Coward, G. D’Agostini, J. Dainton, P. Dalpiaz, H. Danielsson, N. De Simone, D. Di Filippo, L. Di Lella, N. Doble, B. Dobrich, F. Duval, V. Duk, J. Engelfried, T. Enik, N. Estrada-Tristan, V. Falaleev, R. Fantechi, V. Fascianelli, L. Federici, S. Fedotov, A. Filippi, M. Fiorini,J. Fry, J. Fu, A. Fucci, L. Fulton, E. Gamberini, L. Gatignon, G. Georgiev, S. Ghinescu, A. Gianoli, M. Giorgi, S. Giudici, F. Gonnella, E. Goudzovski, C. Graham, R. Guida, E. Gushchin, F. Hahn, H. Heath, T. Husek, O. Hutanu, D. Hutchcroft,L. Iacobuzio, E. Iacopini, E. Imbergamo, B. Jenninger, K. Kampf, V. Kekelidze, S. Kholodenko, G. Khoriauli, A. Khotyantsev, A. Kleimenova, A. Korotkova, M. Koval, V. Kozhuharov, Z. Kucerova, Y. Kudenko, J. Kunze, V. Kurochka, V.Kurshetsov, G. Lanfranchi, G. Lamanna, G. Latino, P. Laycock, C. Lazzeroni, M. Lenti, G. Lehmann Miotto, E. Leonardi, P. Lichard, L. Litov, R. Lollini, D. Lomidze, A. Lonardo, P. Lubrano, M. Lupi, N. Lurkin, D. Madigozhin, I. Mannelli, G. Mannocchi, A. Mapelli, F. Marchetto, R. Marchevski, S. Martellotti, P. Massarotti, K. Massri, E. Maurice, M. Medvedeva, A. Mefodev, E. Menichetti, E. Migliore, E. Minucci, M. Mirra, M. Misheva, N. Molokanova, M. Moulson, S. Movchan, M. Napolitano, I. Neri, F. Newson, A. Norton, M. Noy, T. Numao, V. Obraztsov, A. Ostankov, S. Padolski, R. Page, V. Palladino, C. Parkinson, E. Pedreschi, M. Pepe, M. Perrin-Terrin, L. Peruzzo, P. Petrov, F. Petrucci, R. Piandani, M. Piccini, J. Pinzino, I. Polenkevich, L. Pontisso, Yu. Potrebenikov, D. Protopopescu, M. Raggi, A. Romano, P. Rubin, G. Ruggiero, V. Ryjov,A. Salamon, C. Santoni, G. Saracino, F. Sargeni, V. Semenov, A. Sergi, A. Shaikhiev, S. Shkarovskiy, D. Soldi, V. Sougonyaev, M. Sozzi, T. Spadaro, F. Spinella, A. Sturgess, J. Swallow, S. Trilov, P. Valente, B. Velghe, S. Venditti, P. Vicini, R. Volpe, M. Vormstein, H. Wahl, R. Wanke, B. Wrona, O. Yushchenko, M. Zamkovsky, A. Zinchenko. 


\section{Introduction}

The $K^{+} \rightarrow \pi^{+} v \bar{v}$ and $K_{L} \rightarrow \pi^{0} v \bar{v}$ are flavour changing neutral current decays proceeding through box and electroweak penguin diagrams. A quadratic GIM mechanism and a strong Cabibbo suppression make these processes extremely rare. Using the value of tree-level elements of the Cabibbo-Kobayashi-Maskawa (CKM) triangle, the Standard Model (SM) predicts [1, 2]:

$$
B R\left(K^{+} \rightarrow \pi^{+} v \bar{v}\right)=(8.4 \pm 1.0) \times 10^{-11} \quad B R\left(K_{L} \rightarrow \pi^{0} v \bar{v}\right)=(3.4 \pm 0.6) \times 10^{-11} .
$$

The knowledge of $V_{c b}, V_{u b}$ and the CKM angle $\gamma$ dominate the uncertainties. The theoretical accuracy, instead, is at percent level because: short distance physics dominates due to the top quark loop exchange; the hadronic matrix elements cancel in the normalization of the $\pi v v$ branching ratios (BR) to the measured $K^{+} \rightarrow \pi^{0} e^{+} v$ BR. The dependence on the CKM elements partially cancels in the correlation between $K^{+} \rightarrow \pi^{+} v \bar{v}$ and $K_{L} \rightarrow \pi^{0} v \bar{v}$. Therefore simultaneous measurements of the two BRs' would allow a theoretical clean exploitation of the CKM triangle using kaons only.

The $\pi v v$ decays are very sensitive to physics beyond the SM, probing the highest mass scales among the rare meson decays $[3,4,5,6,7]$. Despite the strong constraints on new physics from LHC, significant modifications of the SM values of the $\pi v v$ BRs' induced by new physics at mass scales up to $100 \mathrm{TeV}$ are possible providing BRs' measurements with $10 \%$ precision at least.

The experimental status is $[8,9,10]$ :

$$
B R\left(K^{+} \rightarrow \pi^{+} v \bar{v}\right)_{\exp }=\left(17.3_{-10.5}^{+11.5}\right) \times 10^{-11} \quad B R\left(K_{L} \rightarrow \pi^{0} v \bar{v}\right)_{\exp }<2.6 \times 10^{-8} 90 \% C L .
$$

The charged mode has been observed by stopping kaon experiments. Still 3 order of magnitude need to be covered to observe the neutral mode.

\section{NA62 experiment at CERN}

The NA62 experiment at CERN $[11,12,13]$ aims to measure the $K^{+} \rightarrow \pi^{+} v \bar{v}$ BR with $10 \%$ precision. Therefore it needs to collect about $10^{13}$ kaon decays using $400 \mathrm{GeV} / \mathrm{c}$ protons from SPS for a $10 \%$ signal acceptance. Keeping the background to signal ratio about $10 \%$ requires the use of almost independent experimental techniques to suppress unwanted final states. With a single event sensitivity of $10^{-12}$ NA62 can afford also a broader physics program [14]. NA62 is running with the apparatus fully operational since 2016.

NA62 adopts a kaon decay-in-flight technique. Fig. 1 shows a schematic view of the apparatus. Primary SPS protons strike a target from which a secondary charged hadron beam of $75 \mathrm{GeV} / \mathrm{c}$ and $1 \%$ momentum bite is selected and transported to the decay region. The detailed descriptions of the apparatus can be found in [13]. The incoming kaon is positively identified by a differential Cerenkov counter (KTAG) and its momentum and direction are measured by three stations of $\mathrm{Si}$ pixel detectors (GTK). About $6 \%$ of beam particles are $K^{+}$. A guard ring detector (CHANTI) vetoes beam inelastic interactions occurring in GTK. A decay tank, holding a $10^{-6}$ mbar vacuum, is surrounded by lead-glass annular calorimeters (LAV) designed to catch photons up to $50 \mathrm{mrad}$. Four stations of straw chambers (STRAW) in vacuum trace downstream charged particles, with a dipole magnet providing a $270 \mathrm{MeV} / \mathrm{c}$ transverse kick for momentum analysis. A RICH counter 


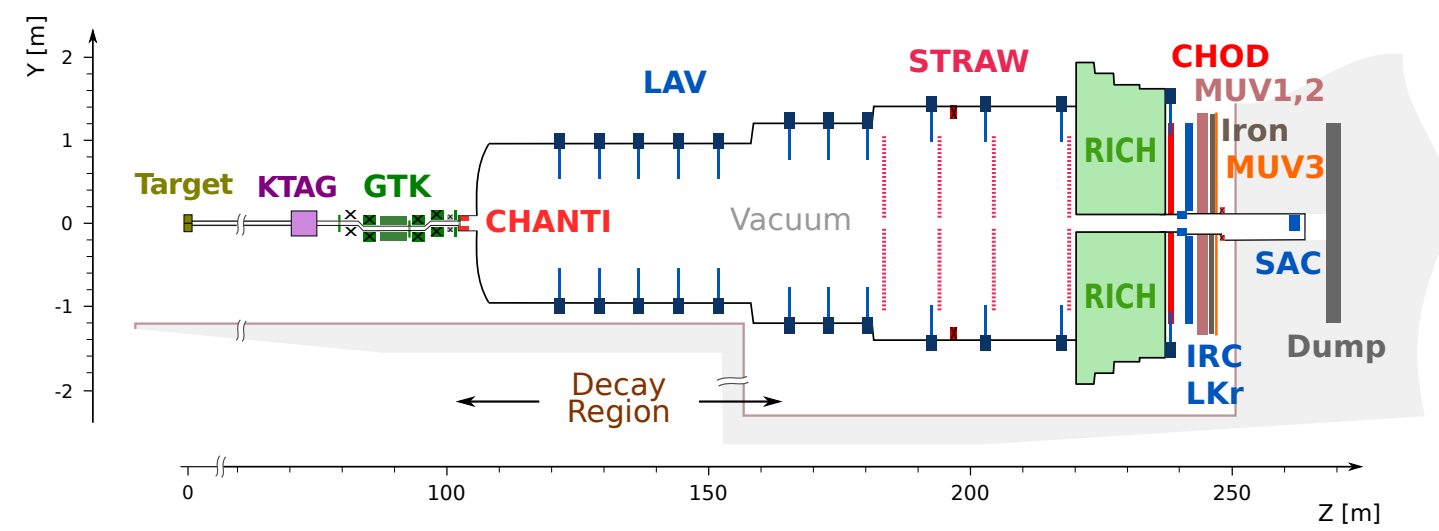

Figure 1: Schematic layout of the NA62 experiment in the $y z$ plane.

time-stamps and identifies charged particles; plastic scintillators (CHOD) are used for triggering and timing. Photon rejection in the forward region is provided by: an electromagnetic calorimeter at liquid krypton (LKr); small angle calorimeters (IRC and SAC). Hadron calorimeters (MUV1,2) and a plastic scintillator detector (MUV3) are used to suppress muons. The SPS delivers $3.3 \times$ $10^{12}$ protons per pulse at full intensity to NA62, corresponding to $750 \mathrm{MHz}$ particle rate in GTK. Information from CHOD, RICH, MUV3 and $\mathrm{LKr}$ are built up online to issue level zero trigger conditions. Software-based variables from KTAG, CHOD, LAV and STRAW provide higher level trigger requirements. $K^{+} \rightarrow \pi^{+} v \bar{v}$-triggered data are taken concurrently with downscaled samples of data for rare kaon decays studies and minimum bias.

The NA62 apparatus has been commissioned in 2015 and 2016. Low intensity data have been taken in 2015 with a minimum bias trigger to study detector performances and to perform physics analysis. In fall 2016 NA62 has collected about $4.5 \times 10^{11}$ kaon decays for $K^{+} \rightarrow \pi^{+} v \bar{v}$ at 20-40\% of nominal intensity. A 4-months run dedicated to $K^{+} \rightarrow \pi^{+} v \bar{v}$ has taken place in 2017 at 50-60\% of the nominal intensity and another one is scheduled in 2018 .

\subsection{Principle of the $K^{+} \rightarrow \pi^{+} v \bar{v}$ Measurement}

The signature of the signal is one track in the initial and one in final state. The main kinematic variable is $m_{\text {miss }}^{2} \equiv\left(P_{K}-P_{\pi^{+}}\right)^{2}$, with $P_{K}$ and $P_{\pi^{+}}$4-momenta of $K^{+}$and of the charged decay product under the $\pi^{+}$mass hypothesis, respectively. Fig. 2 shows the $m_{\text {miss }}^{2}$ theoretical shape of the most important $K^{+}$decay modes compared to $K^{+} \rightarrow \pi^{+} v \bar{v}$. Signal regions are defined between $K^{+} \rightarrow \mu^{+} v$ and $K^{+} \rightarrow \pi^{+} \pi^{0}$ and between $K^{+} \rightarrow \pi^{+} \pi^{0}$ and $K^{+} \rightarrow \pi^{+} \pi^{+} \pi^{-}$. Non gaussian $m_{\text {miss }}^{2}$ resolution tails, radiative tails, $K^{+}-\pi^{+}$mis-matching and neutrinos in final states are the mechanisms inducing backgrounds from $K^{+}$decays entering signal regions. Kinematics, timing, particle identification and photon rejection contribute to background suppression. The analysis is done with $P_{\pi^{+}}$between 15 and $35 \mathrm{GeV} / \mathrm{c}$, so that $\pi^{0} \mathrm{~s}$ ' from $K^{+} \rightarrow \pi^{+} \pi^{0}$ can be hardly missed because of high energy.

\section{$2.2 K^{+} \rightarrow \pi^{+} v \bar{v}$ Analysis}

In this section a preliminary analysis of a 5\% sub-sample of the 2016 data is described. The 


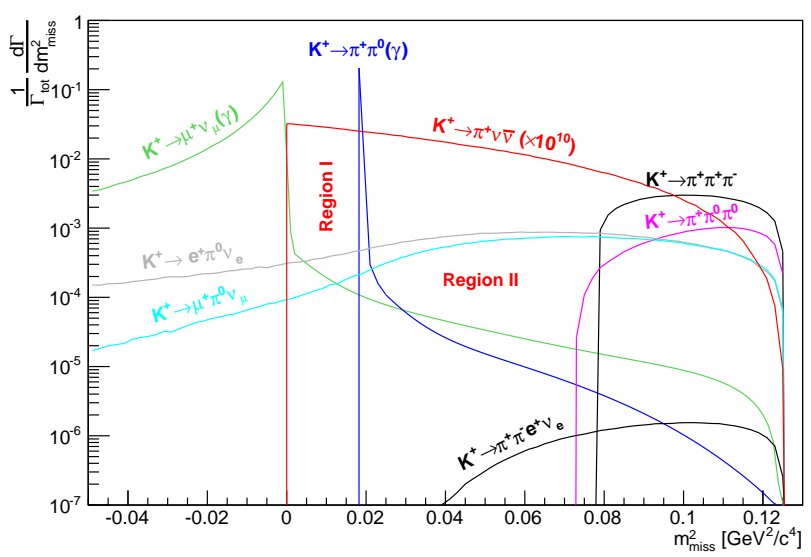

Figure 2: $m_{\text {miss }}^{2}$ shapes for signal and backgrounds of the main $K^{+}$decay modes: the backgrounds are normalized according to their branching ratio; the signal is multiplied by a factor $10^{10}$.

parent $\mathrm{K}^{+}$track is reconstructed and time-stamped in GTK with 100 ps resolution; the daughter $\pi^{+}$track is reconstructed in STRAW. CHOD and RICH measure $\pi^{+}$time with resolution below $100 \mathrm{ps}$. The pion is associated in time to a KTAG kaon signal. The timing and the closest distance of approach between GTK and STRAW tracks allow a precise $K^{+}-\pi^{+}$matching. The kaon mistagging probability at $40 \%$ of nominal intensity is below $2 \%$, signal acceptance about $75 \%$.

Decays are selected within a $50 \mathrm{~m}$ fiducial region beginning $10 \mathrm{~m}$ downstream to the last GTK station (GTK3) to reject events originated from interactions of beam particles in GTK and kaon decays upstream of GTK3. Fig. 3 (left) exemplifies the kinematics of the selected events. The resolution of $m_{\text {miss }}^{2}$ drives the choice of the boundaries of the signal regions. Reconstruction tails from $K^{+} \rightarrow \pi^{+} \pi^{0}, K^{+} \rightarrow \mu^{+} v$ and $K^{+} \rightarrow \pi^{+} \pi^{+} \pi^{-}$set the level of background in signal regions. To reduce it, signal regions are restricted to boxes within a 3D space, defined by the $m_{\text {miss }}^{2}$, the same quantity computed using the momentum of the particle measured by the RICH under $\pi^{+}$hypothesis $\left(m(R I C H)_{m i s s}^{2}\right)$ and computed replacing the 3-momentum of the kaon measured by the GTK with the nominal 3-momentum of the beam $\left(m(N o-G T K)_{m i s s}^{2}\right)$. The probability for $K^{+} \rightarrow \pi^{+} \pi^{0}\left(K^{+} \rightarrow \mu^{+} v\right)$ to enter the signal regions is $6 \times 10^{-4}\left(3 \times 10^{-4}\right)$, as measured on data.

Calorimeters and RICH separate $\pi^{+}, \mu^{+}, e^{+}$. A multivariate analysis combines calorimetric information and provides $10^{5} \mu^{+}$suppression and $80 \% \pi^{+}$efficiency. RICH quantities are used to infer particle types, giving $10^{2} \mu^{+}$suppression and $80 \% \pi^{+}$efficiency. The two methods are independent and therefore able to suppress $\mu^{+}$by 7 orders of magnitude while keeping $65 \%$ of $\pi^{+}$.

Left over events after $\pi^{+}$identification are primarily $K^{+} \rightarrow \pi^{+} \pi^{0}$. Photon rejection exploiting timing coincidences between $\pi^{+}$and calorimetric deposits suppresses them further. The resulting $\pi^{0}$ rejection inefficiency is $(1.2 \pm 0.2) \times 10^{-7}$, as measured from minimum bias and $K^{+} \rightarrow \pi^{+} v \bar{v}$ triggered events before and after $\gamma$ rejection, respectively. Random losses are in the 15-20\% range.

A sample of $K^{+} \rightarrow \pi^{+} \pi^{0}$ from minimum bias is used for normalization. About $0.064 K^{+} \rightarrow$ $\pi^{+} v \bar{v}$ events are expected over $2.3 \times 10^{10} K^{+}$decays. Fig. 3 (right) shows the distribution of residual events in the $m(R I C H)_{m i s s}^{2}$ versus $m_{m i s s}^{2}$ plane. Backgrounds from $K^{+} \rightarrow \pi^{+} \pi^{0}, K^{+} \rightarrow \mu^{+} v$ and $K^{+} \rightarrow \pi^{+} \pi^{+} \pi^{-}$are $0.024,0.011$ and 0.017 , respectively. They are estimated directly from events outside signal regions, with the measured kinematic tails used for extrapolation in signal regions. 

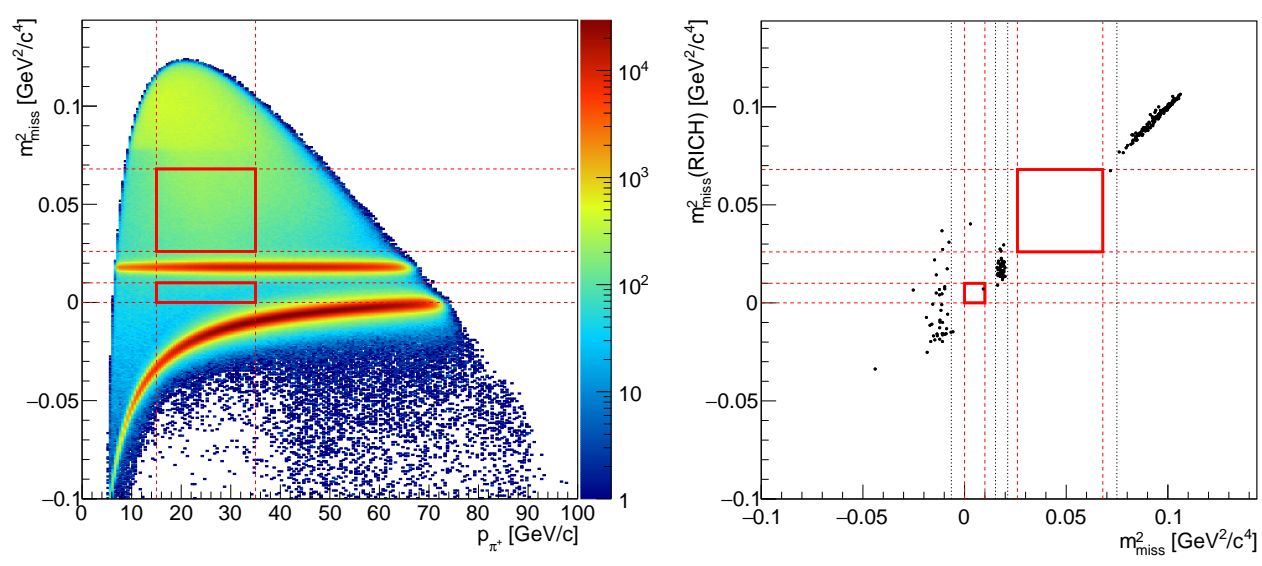

Figure 3: Distribution of $m_{\text {miss }}^{2}$ as a function of track momentum for events selected on minimum bias data; the bands corresponding to $K^{+} \rightarrow \pi^{+} \pi^{0}$ and $K^{+} \rightarrow \mu^{+} v$ decays are clearly visible; the signal regions (red box) are drawn for reference (left). Distribution in the ( $\left.m_{\text {miss }}^{2}(R I C H), m_{\text {miss }}^{2}\right)$ plane of $K^{+} \rightarrow \pi^{+} v \bar{v}$-triggered events passing the selection, except for the cut on $m_{\text {miss }}^{2}(N o-G T K)$; signal regions (red tick boxes) and lines defining background regions (light dashed lines) are drawn; the event in region 1 has $m_{\text {miss }}^{2}(\mathrm{No}-\mathrm{GTK}$ ) outside the signal region (right).

Simulations studies indicate that background from other processes is lower or negligible. The analysis is still on-going together with an optimization of the selection to further reduce backgrounds and increase signal acceptance. No events are observed in signal regions.

NA62 aims to reach the SM sensitivity from the analysis of the 2016 data and to select some tens of $K^{+} \rightarrow \pi^{+} v \bar{v}$ events from the analysis of the data taken in 2017 and expected in 2018. Data taken after 2018 is being considered to complete the measurement.

\section{KOTO experiment at J-PARC}

KOTO [15] is a fixed target experiment at J-PARC [16] in Japan using protons from the Main Ring accelerator [17], aiming to observe few SM $K_{L} \rightarrow \pi^{0} v \bar{v}$ events.

A schematic view of the KOTO detectors is shown in fig. 4. Primary protons from the main ring $(30 \mathrm{GeV} / \mathrm{c})$ impinge on a target with 16 degree production angle. A secondary neutral hadron beam of $1.4 \mathrm{GeV} / \mathrm{c}$ peak momentum, composed by $K^{0}$, neutrons and photons is transported to a $3 \mathrm{~m}$ long decay region holding a vacuum of about $5 \times 10^{-7}$ mbar. Photons detectors hermetically surround the decay volume. The entrance region of the decay volume is designed to minimize beam interactions in the collimators. Scintillator-lead sampling calorimeters at high angles provide photon rejection. A high-resolution electromagnetc calorimeter with crystals of CsI detects forward photons to reconstruct the kinematics of the signal $\pi^{0}$. The small angle region at the exit of the decay volume around the beam pipe is instrumented with plastic counters to veto mainly background from events with charged particles in final state. All the detectors are equipped with waveform digitizers readout to separate signals from pileup.

The first KOTO run took place in 2013 at an intensity of about $2 \times 10^{13}$ proton per pulse, corresponding to $25 \mathrm{~kW}$ power delivered by JPARC. Then KOTO ran in 2015 and 2016 at an 


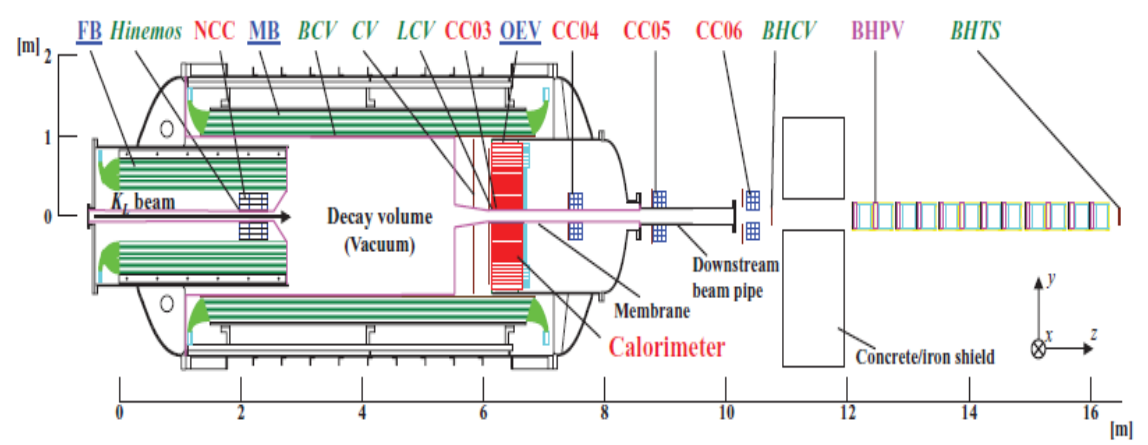

Figure 4: Schematic layout of the KOTO experiment in the $x z$ plane.

intensity of 30-40 kW, collecting about 20 times the statistics of 2013.

\subsection{Principle of the $K_{L} \rightarrow \pi^{0} v \bar{v}$ Measurement}

The signature of the signal is $2 \gamma \mathrm{s}^{\prime}$ in the forward calorimeter. Under the $\pi^{0}$ mass hypothesis both the longitudinal coordinate of the decay vertex $\left(Z_{v t x}\right)$ and the transverse momentum of the $\pi^{0}\left(p_{T}\right)$ can be determined, allowing the identification of a signal region (fig. 5). Rejection of backgrounds from $K_{L} \rightarrow \pi^{0} \pi^{0}\left(\pi^{0}\right)$ fully relies upon photon veto efficiency. Left over events mainly come from $K_{L} \rightarrow \pi^{+} \pi^{-} \pi^{0}$ and neutron induced backgrounds originated from scattering and $\pi^{0}$ production at the entrance. Suppression of these backgrounds depends on the design of the entrance collimators, on the downstream plastic scintillators, on the shape of the calorimetric energy depositions of $\gamma$ and neutron and on the distribution in the $Z_{v t x}$ versus $p_{T}$ plane.

\subsection{3 data analysis and result}

KOTO has analyzed the full 2013 data set, corresponding to about $2.3 \times 10^{11} K_{L}$ decays reaching a single event sensitivity (SES) of $1.3 \times 10^{-8}$. The number of expected events in signal region after the selection is $0.34 \pm 0.16$. One is observed, as shown in fig. 5 (right), leading to an upper limit on the $K_{L} \rightarrow \pi^{0} v \bar{v}$ branching ratio $B R\left(K_{L} \rightarrow \pi^{0} v \bar{v}\right)<5.1 \times 10^{-8}$ at $90 \%$ C.L. [18]. The result is limited by backgrounds from neutron scattering, $K_{L} \rightarrow \pi^{0} \pi^{0}$ and $K_{L} \rightarrow \pi^{+} \pi^{-} \pi^{0}$ to a lesser extent. The background is estimated using simulation overlaid with accidental hits collected with data and validated with $K_{L} \rightarrow \pi^{0} \pi^{0}\left(\pi^{0}\right)$ samples. The decay $K_{L} \rightarrow \pi^{0} \pi^{0}$ is used for normalization. Systematic uncertainties come mainly from the evaluation of the acceptances both for signal and normalization and are taken into account in the final result. Despite the limit is almost two times worse than the best one obtained by the E931 experiment, this analysis has set the level of the sensitivity of KOTO, showing paths for improvements.

\subsection{KOTO after 2013}

A re-design of the entrance region and a better instrumentation of the beam pipe followed the 


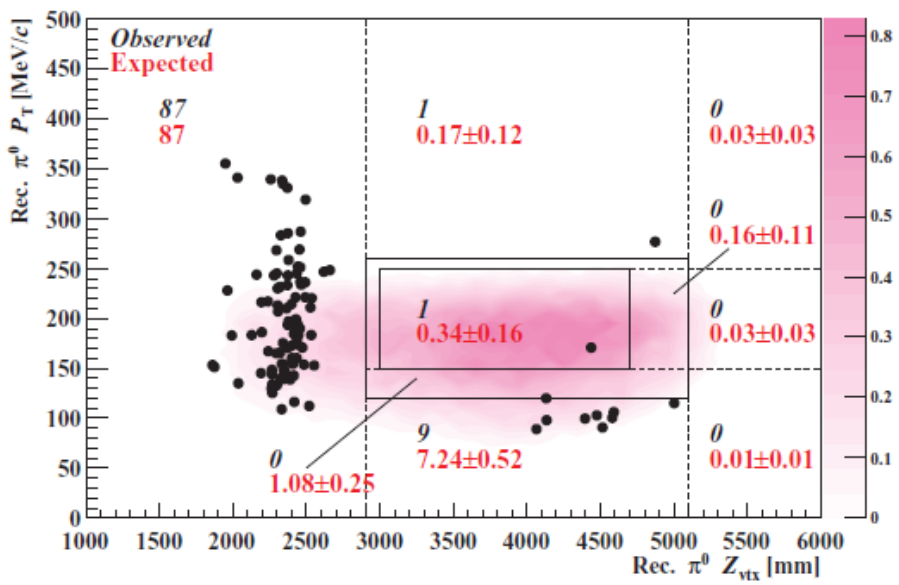

Figure 5: $p_{T}$ versus $Z_{v t x}$ of events with all the analysis cuts imposed. The region surrounded with a thick solid line is the signal region. The black dots represent the data and the contour indicates the distribution of the signal from Monte Carlo simulations. The black italic (red regular) numbers indicate the numbers of observed events (expected background events) for the regions divided by solid and dashed lines

2013 run. A special neutron-enriched run was taken to study the calorimeter response to neutrons, leading to a factor 5 improvement of the neutrons $-\gamma \mathrm{s}^{\prime}$ separation in the CsI calorimeter [20].

A sub-sample of 2015 data comparable in size to the 2013 one is under analysis. The SES and the estimation background in signal region are $5.9 \times 10^{-9}$ and $0.17 \pm 0.05$, respectively; a factor 2 lower than the 2013 analysis thanks to the hardware and software improvements. The 2015 analysis is currently under finalization.

The full 2015 and 2016 data samples correspond to $10^{-9}$ SES, allowing the Grossman - Nir limit [19] for a SM $K_{L} \rightarrow \pi^{0} v \bar{v}$ decay to be reached. However both the data taken so far and the level of background are still not adequate to observe $K_{L} \rightarrow \pi^{0} v \bar{v}$ events. To reach this goal several improvements on the detector side are on progress: a more efficient barrel photon veto installed and used in 2016 [20], modification of the beam pipe to reduce $K_{L} \rightarrow \pi^{+} \pi^{-} \pi^{0}$ background, a dual both-end readout of the CsI calorimeter to better separate neutrons and $\gamma_{\mathrm{s}}$, an increase of the J-PARC power up to $100 \mathrm{~kW}$.

\section{Conclusions}

The kaon experiments NA62 at CERN and KOTO at J-PARC are running to search for physics beyond the SM through the ultra-rare $\pi v v$ decays. NA62 performances are within expectations. The experiment aims to reach the SM sensitivity for $K^{+} \rightarrow \pi^{+} v \bar{v}$ with the analysis of the data collected in 2016 and to get some tens of SM events exploiting data from 2017 and from the already scheduled 2018 run. Data taken after 2018 is being considered to complete the measurement. KOTO experiment is expected to cross the $10^{-9}$ single event sensitivity for $K_{L} \rightarrow \pi^{0} v \bar{v}$ with the data taken in 2015 and 2016. Hardware refurbishments are ongoing aiming to the observation of few SM $K_{L} \rightarrow \pi^{0} v \bar{v}$ events by 2021 . 


\section{References}

[1] J. Brod, M. Gorbahn and E. Stamou, Phys. Rev. D 83 (2011) 034030 [arXiv:1009.0947 [hep-ph]].

[2] A.J. Buras, D. Buttazzo, J. Girrbach-Noe and R. Knegjens, JHEP 1511 (2015) 33 [arXiv:1503.02693 [hep-ph]].

[3] M. Blanke, A.J. Buras and S. Recksiegel, Eur. Phys. J. C 76 no.4 (2016) 182 [arXiv:1507.06316 [hep-ph]].

[4] M. Blanke, A.J. Buras, B. Duling, K. Gemmler and S. Gori, JHEP 903 (2009) 108 [arXiv:0912.3803 [hep-ph]].

[5] A.J. Buras, D. Buttazzo and R. Knegjens, JHEP 1511 (2015) 166 [arXiv:1507.08672 [hep-ph]].

[6] G. Isidori, F. Mescia, P. Paradisi, C. Smith and S. Trine, JHEP 0608 (2006) 64 [hep-ph/0604074].

[7] M. Tanimoto and K. Yamamoto, PTEP 2015 no.5 (2015) 053B07 [arXiv:1503.06270 [hep-ph]].

[8] A.V. Artamonov et al. [E949 Collaboration], Phys. Rev. Lett. 101 (2008) 191802 [arXiv:0808.2459 [hep-ph]].

[9] A.V. Artamonov et al. [E949 Collaboration], Phys. Rev. D 79 (2009) 092004 [arXiv:0903.0030 [hep-ph]].

[10] J.K. Ahn et al. [E391a Collaboration], Phys. Rev. D 81 (2010) 072004 [arXiv:0911.4789 [hep-ph]].

[11] A. Anelli et al., CERN-SPSC-2005-013; SPSC-P-326.

[12] NA62 Technical Design Document NA62-10-07; https://cdsweb.cern.ch/record/14049857.

[13] E. Cortina Gil et al. [NA62 Collaboration], JINST 12 no. 05 (2017) P05025 [arXiv:1703.08501 [hep-ph]].

[14] G. Ruggiero, in proceedings of KAON 2016 conference, J. Phys. Conf. Ser. 800 (2017) no.1 012023.

[15] T. Yamanaka [KOTO Collaboration], PTEP 2012 (2012) 02 B006.

[16] S. Nagamiya, PTEP 2012 (2012) 02B001.

[17] T. Koseki et al., PTEP 2012 (2012) 02B004.

[18] J.K Ahn et al. [KOTO Collaboration], PTEP 2017 no.2 (2017) 021 C01 [arXiv:1609.03637 [hep-ph]].

[19] Y. Grossman and Y. Nir, Phys. Lett. B 398 (1997) 163 [hep-ph/9701313].

[20] K. Shiomi, in proceedings of KAON 2016 conference, J. Phys. Conf. Ser. 800 (2017) no.1 012022. 